\title{
Intracavity optical trapping with Ytterbium doped fiber ring laser
}

Rania Sayed

Fatemeh Kalantarifard

Parviz Elahi

F. Omer llday

Giovanni Volpe

Onofrio M. Maragò 


\title{
Intracavity Optical Trapping with Ytterbium Doped Fiber Ring Laser
}

\author{
Rania Sayed $^{a, b}$, Fatemeh Kalantarifard ${ }^{c}$, Parviz Elahi $^{c}$, F. Omer Ilday ${ }^{c}$, Giovanni Volpe $^{c}$, \\ Onofrio M. Maragó ${ }^{a}$ \\ ${ }^{a}$ Istituto Processi Chimico-Fisici (Messina), Consiglio Nazionale delle Ricerche, Viale F. \\ Stagno D'Alcontres, 37, I-98158 Messina, Italy; ${ }^{b}$ Scuola di Dottorato in Fisica, Universiti \\ Messina, V.le F. Stagno D'Alcontres, 31, I-98158 Messina, Italy; ${ }^{c}$ Department of Physics, \\ Bilkent University, 06800 Ankara, Turkey.
}

\begin{abstract}
We propose a novel approach for trapping micron-sized particles and living cells based on optical feedback. This approach can be implemented at low numerical aperture (NA=0.5, 20X) and long working distance. In this configuration, an optical tweezers is constructed inside a ring cavity fiber laser and the optical feedback in the ring cavity is controlled by the light scattered from a trapped particle. In particular, once the particle is trapped, the laser operation, optical feedback and intracavity power are affected by the particle motion. We demonstrate that using this configuration is possible to stably hold micron-sized particles and single living cells in the focal spot of the laser beam. The calibration of the optical forces is achieved by tracking the Brownian motion of a trapped particle or cell and analysing its position distribution.
\end{abstract}

Keywords: Optical trapping, fiber ring laser, optical feedback, particle tracking, digital video microscopy.

\section{INTRODUCTION}

Arthur Ashkin pioneered the field of laser-based optical trapping (OT) in the early 1970s. In a series of seminal papers, he demonstrated that optical forces could displace and levitate micron-sized dielectric particles in both water and air, ${ }^{1}$ and he developed a stable, three-dimensional trap based on counterpropagating laser beams. This seminal work eventually led to the development of the single-beam gradient force optical trap, ${ }^{2}$ or "optical tweezers" as it has come to be known. Ashkin and co-workers employed optical trapping in a wide-ranging series of experiments from the cooling and trapping of neutral atoms ${ }^{3}$ to manipulating live bacteria and viruses. Today, optical traps continue to find applications in both physics and biology. In parallel with the widespread use of optical trapping, theoretical and experimental work on fundamental aspects of optical trapping is being actively pursued. ${ }^{1,4-11}$ As the early work on optical trapping was made possible by advances in laser technology, much of the recent progress in optical trapping can be attributed to further technological development. The ability of optical tweezer to manipulate micronscale particles precisely and nondestructively has allowed a wide and growing variety of applications in different fields of sciences such as cellular and molecular biology, soft matter, nanotechnology and physics. The ability to apply forces in the range of femto to pico Newton on nanoparticles and to measure their displacements with nanometer precision is crucial for the investigation of colloidal and condensed matter systems. ${ }^{12-16}$ More recently OT have been also used to manipulate, rotate and assemble a variety of nanostructures, such as carbon nanotubes, nanowires, polymer nanofibers, and metal nanoparticles.

In recent years, the capabilities of fiber lasers have made significant progress, achieving high output power, excellent beam quality, compact size, long life time, low maintenance and operational robustness. Fiber lasers have been commercially available for about a decade, but their application has primarily been for research and laboratory applications. With the improvements made in gain fiber and fiber Bragg-grating mirrors, higher powers and therefore more widespread applications are enabled. Fiber lasers are optically pumped, and are typically quite efficient; some continuous wave $(\mathrm{CW})$ designs show efficiency of $75 \%$ or more. Ultimately, any fiber laser design requires pump light, with a wavelength matching the absorption band created by the rare-earth dopant in the fiber. Optical feedback achieved inside a ring cavity fiber laser not only enhances the wavelength tenability, but also reduces the intrinsic linewidth of spectral line.

Optical Trapping and Optical Micromanipulation X, edited by Kishan Dholakia, Gabriel C. Spalding, Proc. of SPIE Vol. 8810, 88102S · @ 2013 SPIE · CCC code: 0277-786X/13/\$18 · doi: 10.1117/12.2022899 


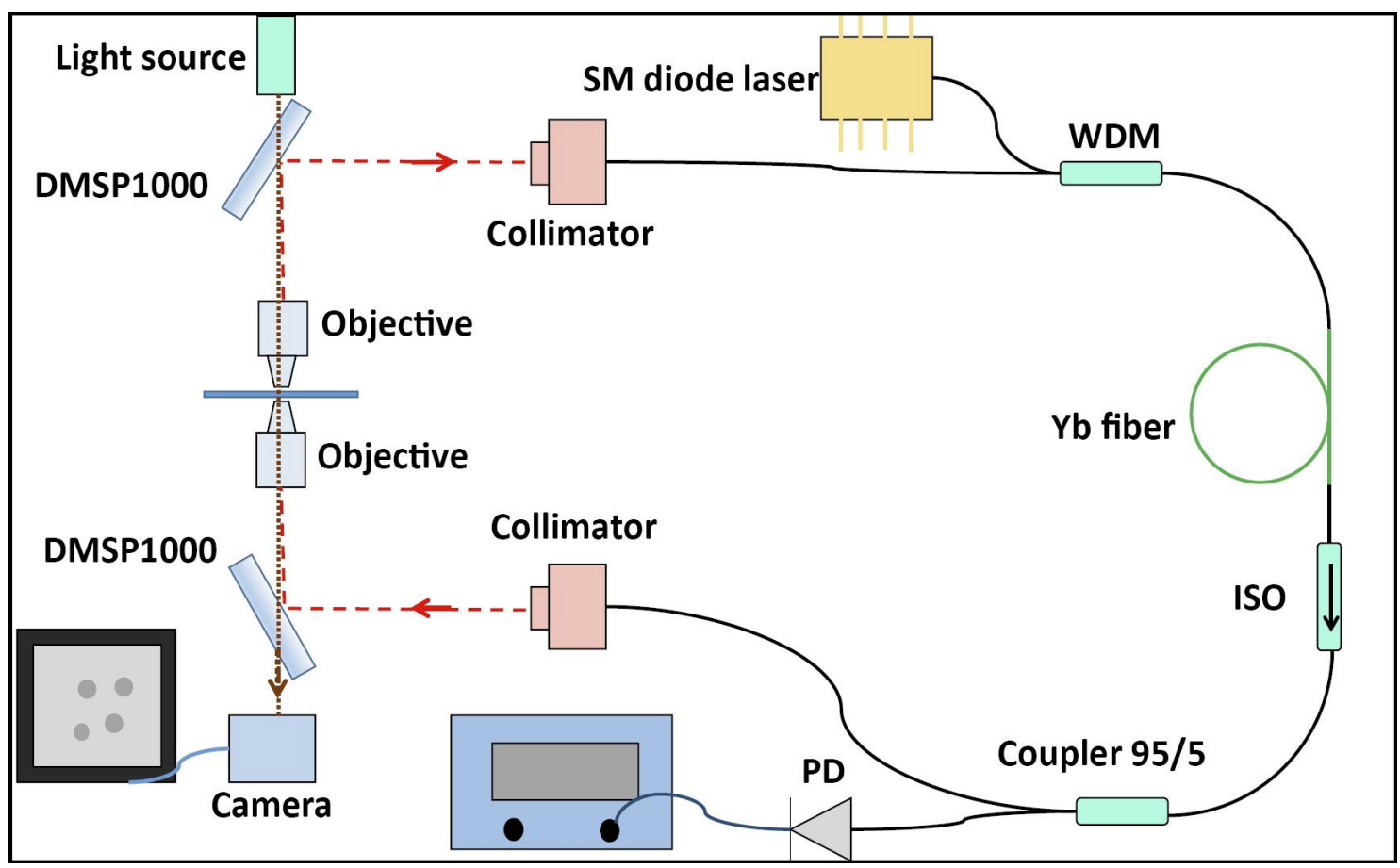

Figure 1. Schematic of the experimental setup. The red dashed line shows the propagation of light in free space. SM diode laser: Single-mode diode laser. ISO: isolator. WDM: wavelength-division multiplexer. PD: photo diode. DMSP1000: Short pass dichroic mirror with cut-off at $1000 \mathrm{~nm}$.

In this paper, we demonstrate a novel technique for optical tweezing by using the optical feedback inside the ring cavity fiber laser. In standard OT high intensity of the laser at the focal spot is often undesirable because of possible photodamage of the trapped sample. Here, the use of optical feedback in a ring cavity fiber laser reduces the intensity of operation of the trap. We demonstrate stable trapping operation employing low numerical aperture objectives $(\mathrm{NA}=0.5)$. In this novel experimental configuration the optical feedback in the cavity of the fiber laser is controlled by the light scattering from the trapped particle. When there is no particle in the trap, the optical feedback increases the trapping power in the focal point above the microscope objective. Instead, when a particle falls in the trap the optical feedback is reduced and the trap works at low power preventing photodamage and relaxing the stringent conditions on high numerical aperture for standard optical tweezers (typically NA 1.3).

\section{LASER SOURCE}

Overview Fiber lasers are recently receiving a more considerable attention due to their outstanding advantages in comparison to solid state lasers. They are more stable, with higher beam quality and more efficient. A configuration in ring cavity is one of the most common cavity for low power fiber lasers. In the ring cavity, a wavelength division multiplexer (WDM) generally is used to combine the pump power with the signal power of the laser. A coupler is employed as a output-coupler and plays the same role as a fiber Bragg grating in line-resonators. Rare earth ions are doped in fibers to produce gain fibers. Er and Yb-doped fibers are among the most well active fibers.

Ring fiber lasers have the ability to provide stable, single-frequency, and extremely narrow-linewidth laser oscillation. Figure 1 shows the experimental set up and the configuration of fiber laser. The isolator in the setup provides for unidirectional operation, which is required for single-longitudinal mode operation. The two most important parameters characterizing a laser are the threshold pump power and the efficiency with which the laser converts the pump power into laser power once it has reached threshold. Laser threshold is determined by requiring that the gain compensate total cavity losses during each round trip. ${ }^{17-19}$ If we consider a Fabry-Perot 
cavity, formed by placing two mirrors of reflectivities $R_{1}$ and $R_{2}$ at the two ends of the gain medium of length $\mathrm{L}$ (R1 and R2 represent the reflectivity of fiber Bragg grating (FBG) for line cavity and can be obtained from the coupling efficient of the coupler in ring cavity) the threshold condition becomes

$$
G^{2} R_{1} R_{2} \exp \left(-2 \alpha_{i n t} L\right)=1
$$

where $\mathrm{G}$ is the single pass amplification factor and $\alpha_{\text {int }}$ denotes all internal losses within the cavity. For a single pass cavity, the output power $P_{\text {out }}$ versus the input power $P_{\text {in }}$ can be written as ${ }^{22}$

$$
P_{\text {out }}=\sigma_{s}\left(P_{\text {in }}-P_{t h}\right)
$$

where $\sigma_{s}$ is the slope efficiency and $P_{t h}$ is the laser threshold power.

By considering $R_{1}=1$ and $R_{2}=1-T$, where $T$ is the transmittance of the output coupler, the slope efficiency is written as

$$
\sigma_{s}=\frac{-\ln (1-T)}{\alpha_{i n t}-\ln (1-T)} \eta
$$

where $\eta$ ia the efficiency factor. ${ }^{22}$

The threshold power of the laser can be obtained by ${ }^{22}$

$$
P_{t h}=\left(\frac{\alpha_{i n t}-\ln (1-T)}{2}\right) \frac{A h \nu_{e}}{\eta} \sigma \tau_{f}
$$

where $A$ is the effective area of the gain medium, $h$ is the Palnck constant, $\nu_{e}$ is the laser frequency, $\tau_{f}$ is the excited state lifetime and $\sigma$ is the stimulated emission cross-section.

Laser construction. Fiber lasers are usually meant to be lasers with optical fibers as gain media. These fibers are placed inside a cavity designed to provide an optical feedback. In most cases, the gain medium is a fiber doped with rare earth ions such as erbium $\mathrm{Er}^{3+}$, neodymium $\mathrm{Nd}^{3+}$ or ytterbium $\mathrm{Yb}^{3+}$. One or several fiber-coupled laser diodes are used for pumping. Therefore, most fiber lasers are diode-pumped lasers. The waveguiding effect and the small effective mode area usually lead to substantially different properties of the lasers. For example, they often operate with much higher laser gain and resonator losses. Ring cavities are often used to realize unidirectional operation of a laser. In the case of fiber lasers, an additional advantage is that a ring cavity can be made without using mirrors, resulting in an all-fiber cavity. In the ring cavity which contain the doped fiber, an isolator is inserted within the loop for unidirectional operation.

A custom CW ring cavity fiber laser with cavity length $5.5 \mathrm{~m}$ of fiber is built at infrared wavelength 1030 $\mathrm{nm}$ and maximum output power $200 \mathrm{~mW}$ as shown in Fig. 2. It has gain fiber doped with ytterbium and is pumped by a butterfly diode laser at wavelength $976 \mathrm{~nm}$ which has a narrow spectral line (about $3 \mathrm{~nm}$ FWHM). The $976 \mathrm{~nm}$ diode has a high absorption band so it doesn't drift away from the pump band over temperature variations which required to maintain wavelength stability. The Pump Laser Protector $(P L P)$ is spliced to the output of the diode laser to filter out any possible light from the optical link that may enter the cavity of the diode laser and affect its operation or even damage it. The output fiber of $P L P$ is fusion spliced to the input fiber of a Wavelength Division Multiplexing $(W D M)$ to multiplex the two different wavelengths $976 \mathrm{~nm}$ and $1030 \mathrm{~nm}$ onto a single optical fiber. The output fiber of WDM is spliced to the input of gain fiber "Ytterbium doped fiber" which consider as active medium of the laser. The output of the gain fiber is fusion spliced to the isolator, which allow to the laser light goes through one direction inside the ring cavity. An output coupler is spliced to the collimator which allows to $95 \%$ of laser light to go again inside the ring cavity and $5 \%$ is used as indicator for the laser power inside the cavity. The intracavity signal is detected by an oscilloscope.

\section{INTRACAVITY OPTICAL TWEEZERS SETUP}

The laser in this experiment is a home built Yb-doped fiber laser (Fig. 2). The setup comprises of a ring cavity fiber laser which the trapping components is a part of the cavity, which is the main difference of this system from 


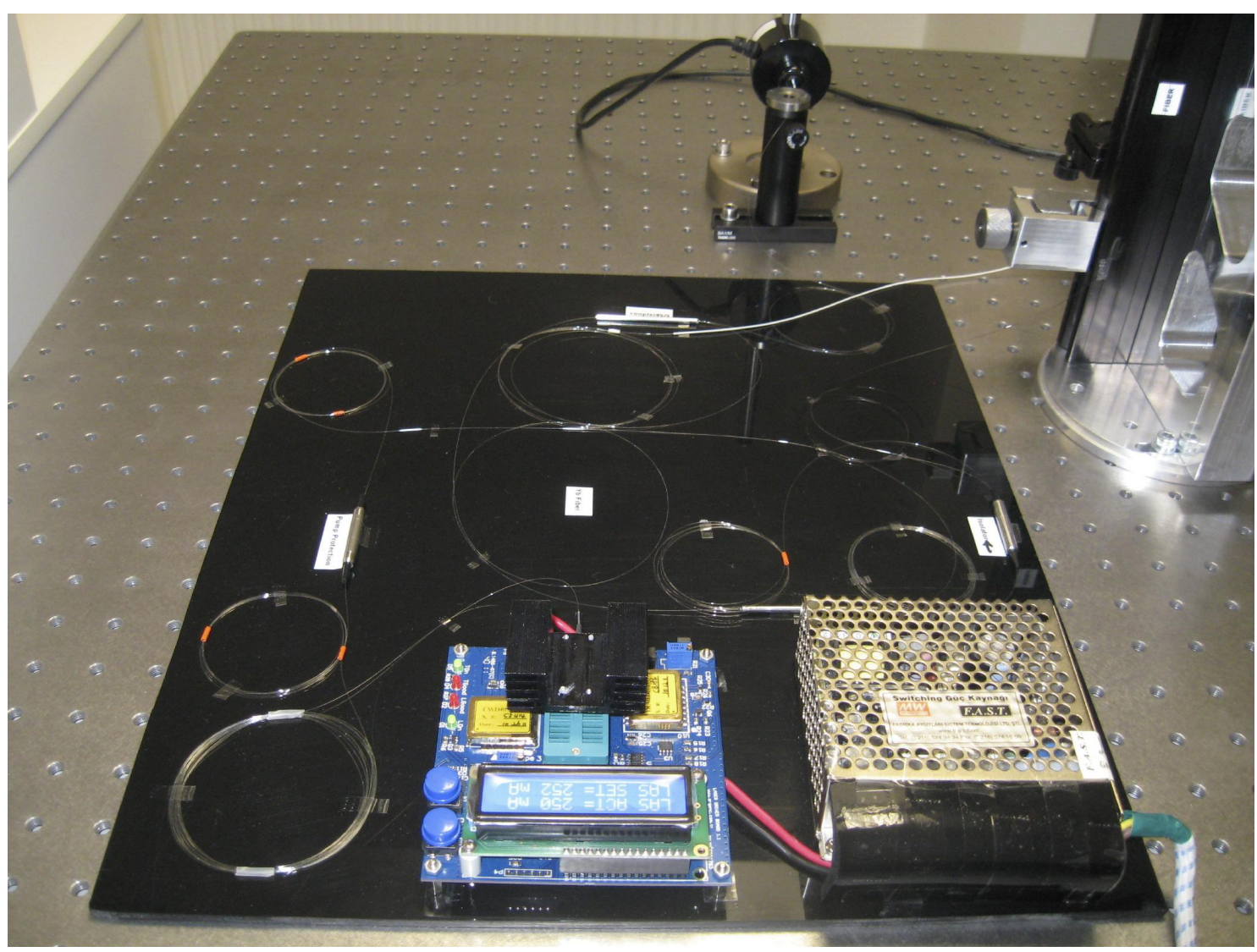

Figure 2. Picture of the $\mathrm{CW}$ ring cavity fiber laser.

conventional extra cavity optical trapping setups. This configuration comprises of a diode-pumped Yb-fiber laser and a free space optics concerns with the trapping. The fiber laser part consists of a single mode diode laser with $976 \mathrm{~nm}$ center wavelength and $3 \mathrm{~nm}$ spectral bandwidth. The output power of diode can be increased up to $600 \mathrm{~mW}$. A wavelength division multiplexer (WDM) combines the diode laser light with the fiber laser signal within the cavity at $1030 \mathrm{~nm}$. The insertion loss of WDM is measured about $0.7 \mathrm{~dB}$. The gain fiber is $20 \mathrm{~cm}-$ long single mode $\mathrm{Yb}$ with $500 \mathrm{~dB}$ gain at $976 \mathrm{~nm}$. The core and clad radius of the gain fiber and other passive fibers is $6 \mu \mathrm{m}$ and $125 \mu \mathrm{m}$, respectively. An inline isolator with $4 \mathrm{~dB}$ insertion loss is employed in order to make an unidirectional propagation within the cavity. By using of $5 \%$ port of a $95 / 5$ coupler followed after isolator, the output power is monitored. A silicon photo diode convert optical signal to electrical signal is measured by an oscilloscope. As seen from the schematic figure, one part of the cavity is free space and comprises of collimators, dichroic mirrors and objectives. The bottom collimator, collimate the output beam toward the dichroic mirror (DM). The dichroic mirror which is used in this setup passed and reflect wavelength beyond and shorter than $1000 \mathrm{~nm}$, respectively. The reflectance of the DM for $1030 \mathrm{~nm}$ which is the center wavelength of the laser system is measured as $90 \%$. The reflected beam then passed through an $20 \mathrm{X}$ objective with $0.5 \mathrm{NA}$ and focused on the sample. The diameter of the spot in the focal point is almost $2 \mu \mathrm{m}$. Another objective then collect the scattered beam from the particle and by using of another dichroic mirror and collimator, leads to the cavity again.

The laser beam is scattered by particle after trapping. By increasing the internal loss of the cavity, the threshold power of the laser is increased (Eq. 4) and the power of the laser then varies.

Video particle tracking. Tracking particles in a digital video stream is a very convenient way to take position measurements, especially as most tweezers systems already feature a camera to observe the sample. Modern cameras can reach frame rates of up to tens of $\mathrm{KHz}$, approaching the speeds attainable with interferometric 

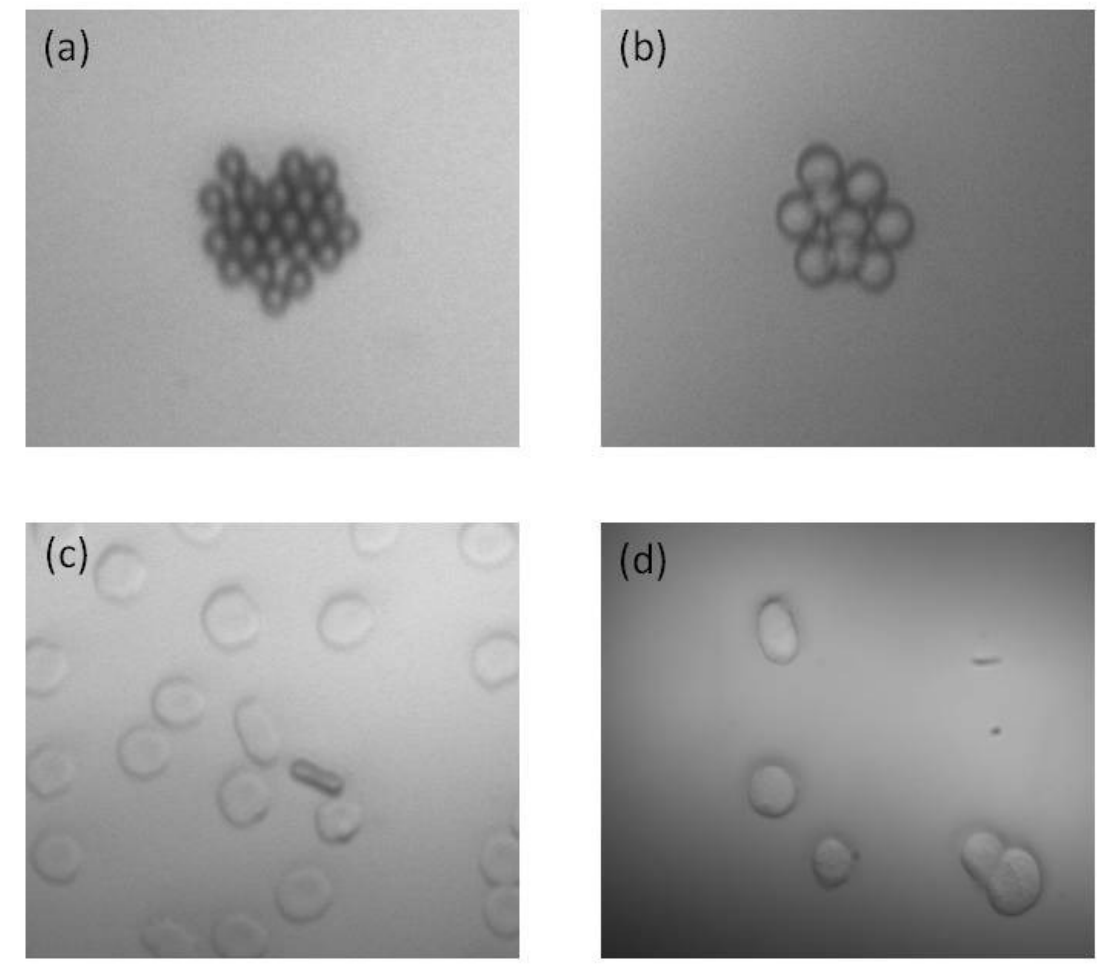

Figure 3. Trapping of particles and cells. (a) Trapping group of melamine particles with diameter $2 \mu$ at power $22 \mathrm{~mW}$. (b) Trapping group of silica particles with diameter $5 \mu \mathrm{m}$ at power $16 \mathrm{~mW}$ (c) Trapping red blood cell with diameter $8 \mu \mathrm{m}$ at power $11 \mathrm{~mW}$ and (d) Trapping cancer cell with diameter $16.5 \mu \mathrm{m}$ at power $30 \mathrm{~mW}$

systems. Even inexpensive cameras can now exceed $1 \mathrm{KHz}$ if the acquisition region is limited to only a few trapped objects, typically a region around 50-120 pixels high and 600 pixels wide can be acquired at $\mathrm{KHz}$ rates on a gigabit Ethernet based camera.

The ability to use a camera simplifies taking force for two dimensional measurements. Once the magnification is known and checked with a ruled slide, camera particle tracking reads out in absolute distance units, eliminating at least one calibration step. It is also linear over the entire field of view of the camera, typically $20-100 \mu \mathrm{m}$. A variety of free and commercial computer programs can be used to track particles on the fly with accuracy much less than a pixel, using the simple "center of mass' algorithm or other more sophisticated techniques. For microscopes used in optical tweezers, this typically results in a resolution of a few nanometers. ${ }^{20}$

\section{RESULTS AND DISCUSSION}

Optical trapping experiments are performed in a liquid environment using calibration particles as silica and melamine microspheres with 2, 4, and $5 \mu \mathrm{m}$ and living cells as blood, cancer and yeast as shown in Fig. 3 . Stiffness constant $K$ of trapped particle is measured at different trapping power and all measurements are carried out at room temperature $\left(22{ }^{\circ} \mathrm{C}\right)$.

Trapping calibration beads. A micro-particle trapped in optical tweezers is unavoidably driven by Brownian force to undertake a three-dimensional random walk around its equilibrium position within a confined volume. The extent of fluctuation in particle's position depends on many factors including laser power, numerical aperture of the focusing beam, the temperature, the viscosity of the surrounding medium, and the size (and the weight) 

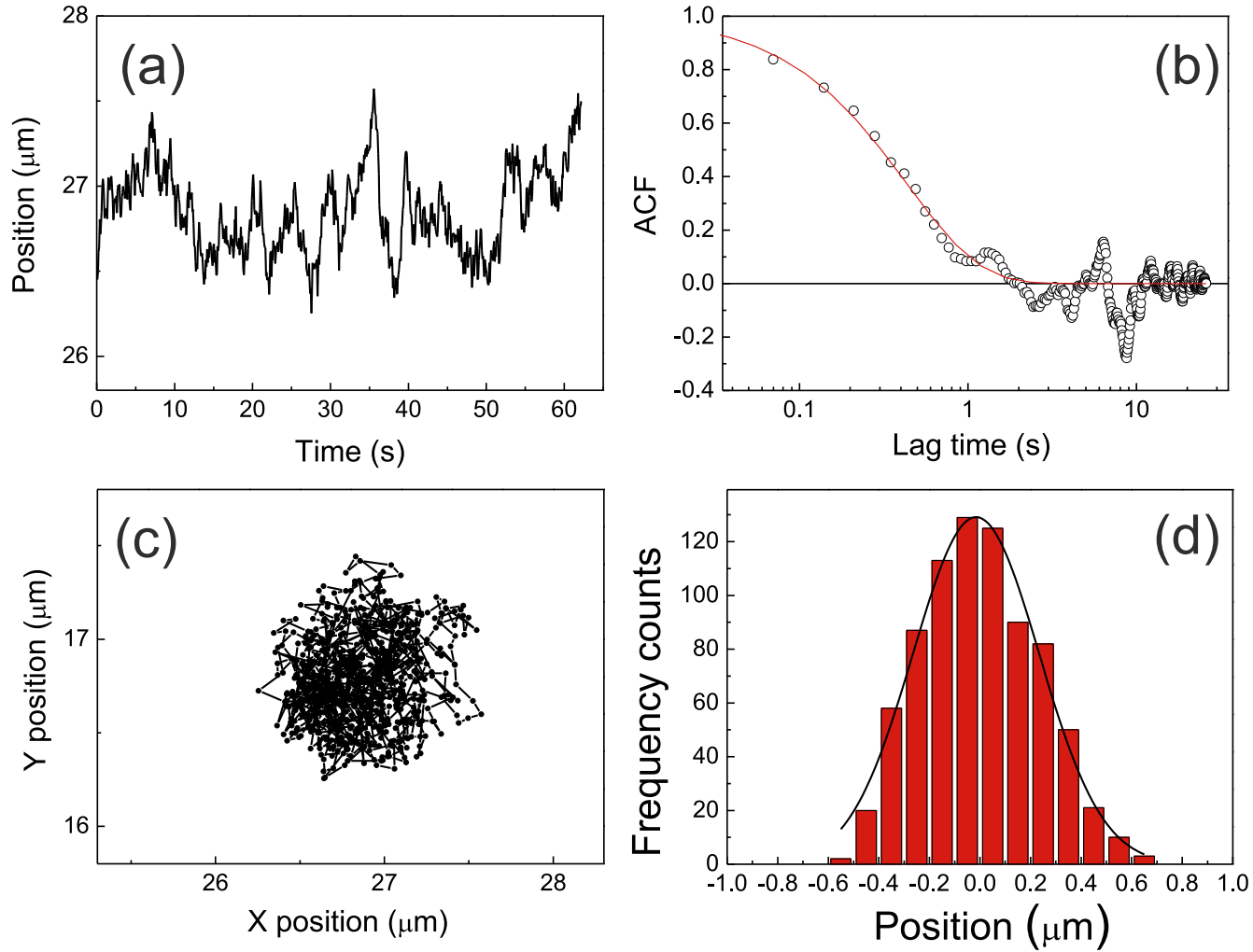

Figure 4. Calibration of optical forces on a silica bead $5 \mu \mathrm{m}$. (a) Fluctuations of trapped particle in X position. (b) Autocorrelation function (ACF) for trapped particle at $30 \mathrm{~mW}$ in $\mathrm{x}$ direction. (c) Trajectory of trapped particle. (d) Histogram of particle fluctuations

of the micro-particle. Under the experimental conditions reported in this chapter the fluctuation of the trapped particle was found to be in the sub-micron to micron range. The particle trajectory can be conveniently tracked by a CCD camera. For each particle trapped in optical tweezers with a specific set of trapping parameters (specified by optical wavelength, optical power, numerical aperture NA, etc.), optical force along each of the 2 dimensions is deduced based on the analysis of the position distribution of the particle.

In our experiments the silica bead with 4 and $5 \mu$ are trapped at current $200 \mathrm{~mA}$ (power at sample is $9.7 \mathrm{~mW}$ ). For the case of $5 \mu \mathrm{m}$ diameter we get the trajectory of particle displacement and autocorrelation function as in fig. (4). When the optical trapping power (the laser power measured at sample) is $30 \mathrm{~mW}$ and the numerical aperture (NA) of the focusing objective lens is $0.5(20 \mathrm{x})$, the corresponding optical force constants along the $\mathrm{x}$ and y directions are determined to be $K_{x}=0.108 \pm 0.0037 \mathrm{PN} / \mu \mathrm{m}$ and $K_{y}=0.098 \pm 0.0072 \mathrm{PN} / \mu \mathrm{m}$. When the optical power of trapping beam increases from $9.7 \mathrm{~mW}$ to $42 \mathrm{~mW}$ the optical force becomes larger and larger as shown in fig. 6 .

Trapping living cells. Optical forces (on the order of a few pico-Newtons to tens of pico-Newtons) in optical tweezers with near infrared (NIR) laser beam at $1.06 \mu$ wavelength were soon demonstrated for non-invasive trapping and manipulation of a single living cell. ${ }^{21}$ With proper force calibration, optical tweezers can be used as a convenient force transducer for the measurement of biological molecular interactions. When the biological cell trapped the optical force constant confining the cell can be measured by tracking and analysing it in 3 dimensions. The force constant may vary significantly from one cell to another even under the same experimental conditions. In this experiment single living cell can be trapped, as shown in Fig. 4 and analysed 

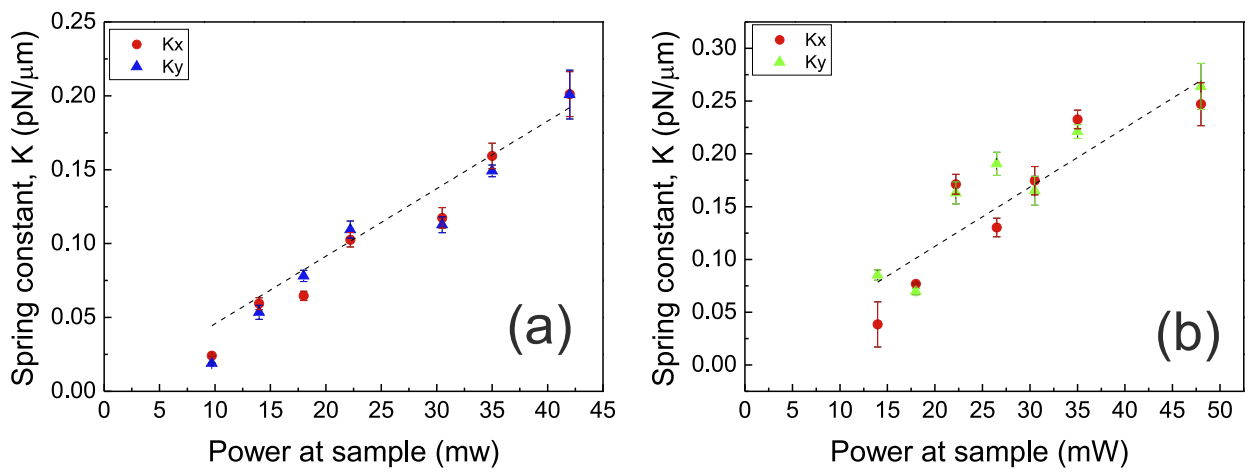

Figure 5. (a) Optical force as a function of laser power at sample for silica bead $5 \mu \mathrm{m}$ in $\mathrm{x}$ and y directions. (b) Optical force as a function of laser power at sample for white blood cell $7.8 \mu \mathrm{m}$ in $\mathrm{x}$ and y directions.

the two dimensional optical force field with the same method as microparticle. Under the same experimental conditions (laser wavelength $1030 \mathrm{~nm}$, output power $72 \mathrm{~mW}, \mathrm{NA}=0.5$ ) the optical forces on the red blood cell and white blood cell are measured separately during increasing the optical power at sample from $10 \mathrm{~mW}$ to 42 mw as shown in fig.7. For sample preparation of white blood cells, fresh blood cells are suspended in a viscous fluid, Dulbecco's Modified Eagle's Medium formulation (DMEM), and Centrifugation is carried out in order to purify plasma from formed elements of the blood including platelets that can be trapped together with red blood cells (RBCs) and preventing correct measurements. The red blood cell is much more sensitive to laser light, it rotates and changes the physical shape during trapping and by increasing the trapping power to $22 \mathrm{~mW}$ the cell die. In contrast the white blood cell can trap at higher power without any change in the shape.

\section{CONCLUSIONS}

To conclude, we have provided a novel technique for optical trapping with a fiber ring laser system based on low numerical aperture objective $(\mathrm{NA}=0.5 / 20 \mathrm{X})$. By this technique we trap calibration beads $4,5 \mu \mathrm{m}$ and single living cell as yeast, blood, cancer cell. Intracavity optical trapping with low numerical aperture lenses increases trapping depth, efficiency and spatial range in experiments holding promises for applications on biological systems that are affected by photodamage.

\section{REFERENCES}

1. A. Ashkin, "Acceleration and Trapping of Particles by Radiation Pressure," Phys. Rev. Lett. 24, 156-159 (1970).

2. A. Ashkin et al., "Observation of a single-beam gradient force optical trap for dielectric particles," Opt. Lett. 11, 288-290 (1986).

3. S. Chu et al., "Experimental Observation of Optically Trapped Atoms," Phys. Rev. Lett. 57, 314-317 (1986).

4. A. Ashkin, "History of optical trapping and manipulation of small-neutral particle, atoms, and molecules," IEEE J. Sel. Top. Quantum Electron 6, 841-856 (2000).

5. A. Ashkin, "Optical trapping and manipulation of neutral particles using lasers," Proc. Natl. Acad. Sci. U. S. A. 94, 4853-4860 (1997).

6. D. V. McGloin et al., "Interfering Bessel beams for optical micromanipulation," Opt. Lett. 28, 657-659 (2003).

7. M. P. MacDonald et al., "Creation and Manipulation of Three-Dimensional Optically Trapped Structures," Science 296, 1101-1103 (2002).

8. L. Paterson et al., "Controlled Rotation of Optically Trapped Microscopic Particles," Science 292, 912-914 (2001). 
9. V. Garces-Chavez et al., "Simultaneous micromanipulation in multiple planes using a self-reconstructing light beam," Nature 419, 145-147 (2002).

10. G. Pesce et al., "Quantitative assessment of non-conservative radiation forces in an optical trap." $E P L$ (Europhys. Lett.) 86, 38002 (2009).

11. V. Garbin et al., "Mie scattering distinguishes the topological charge of an optical vortex: a homage to Gustav Mie." New J. Phys. 11, 013046 (2009).

12. O. M. Maragò et al., "Femtonewton Force Sensing with Optically Trapped Nanotubes," Nano Lett. 8 3211-3216 (2008).

13. A. Irrera et al., "Size-Scaling in Optical Trapping of Silicon Nanowires," Nano Lett. 11, 4879-4884 (2011).

14. M. G. Donato et al., "Optical trapping of nanotubes with cylindrical vector beams," Opt. Lett. 37, 33813383 (2012).

15. G. Volpe, D. Petrov, "Torque Detection using Brownian Fluctuations," Phys. Rev. Lett. 97, 210603 (2006)

16. G. Volpe et al., "Brownian motion in a nonhomogeneous force field and photonic force microscope." Phys. Rev. E 76, 061118 (2007).

17. O. Svelto, D. C. Hanna, Principles of Lasers, Plenum, New York, 4th Ed. (1998).

18. D. C. Hanna et al., "Frequency upconversion in Tm- and Yb:Tm-doped silica fibers," Opt. Commun. 78, 187-194 (1990).

19. J. Y. Allain et al., "Tunable CW lasing around 610, 635, 695, 715, 885 and 910 nm in praseodymium-doped fluorozirconate fibre," Electron. Lett. 27, 189-191 (1991).

20. M. Padgett, R. Bowman, "Tweezers with a twist," Nature Photon. 5, 343-348 (2011).

21. A. Ashkin et al., "Optical trapping and manipulation of single cells using infrared laser beam," Nature 330, 769-771 (1987).

22. W. Koechner, Solid-state laser engineering, Springer, 6th Ed. (2006). 